\title{
The Development and Current Status of Fracture Mechanics
}

\author{
Ting-Ting LI ${ }^{a}$, Li-Jun OU-YANG ${ }^{b}$, Bin ZHEN ${ }^{c,}$ \\ School of Environment and Architecture, University of Shanghai for Science and Technology, \\ Shanghai 200093, China \\ alitingitnghb@163.com, bouyanglijun82@163.com, 'zhenbin80@163.com \\ "Corresponding author
}

Keywords: Fracture Mechanics, Fracture Criteria, Test Standards.

\begin{abstract}
Fracture mechanics, began in the 1950s, is a new branch of solid mechanics. In this paper, we first talk about the history of fracture mechanics at home and abroad. Then the basic theory of fracture mechanics is briefly described in the second part, in which the basic theory and fracture criteria of the three kinds of classical fracture mechanics theories, linear elastic fracture mechanics, elastic-plastic fracture mechanics and fracture dynamics, are emphatically introduced. After this, some current test standards are listed. In the end, certain problems to be solved and the development prospects of fracture mechanics are pointed out.
\end{abstract}

\section{Introduction}

During and after the World War II, the fracture accident of free wheel, United States's all welded steel ship, took place more than a thousand times. Studies of low stress brittle fracture accidents like free wheel's accident give birth to "fracture mechanics".

Fracture mechanics and the general understanding of "fracture" in mechanics are totally two different concepts. One case we know about the general fracture phenomenon is the tensile fracture of a test rod. It's well agreed that whether a test rod occurs tensile fracture is determined by the the magnitude relation between tensile stress and the limit strength of materials. However, free wheel's steel plate hull fracture occurred in the hatch point, first with crack, then the brittle fracture. Traditional strength design methods such as permissible stress method can not take the effect of cracks into account, as the stress concentration at the crack tip makes theoretical solution approach the infinite value.

As a branch of solid mechanics, the task of fracture mechanics is to find how the crack arises, grows, and makes the component with initial cracks fracture under the action of loads.

\section{The Development of Fracture Mechanics at Home And Abroad}

\section{The Development of Fracture Mechanics in Foreign Countries}

Elasticity theory is the theoretical basis of fracture mechanics. In 1913, Inglis [1] solved the tensile stress problem of infinite plane with a elliptical hole in elliptic coordinate system and suggested that stress concentration at the end of the crack is infinite. In 1939, Westergaar [2] directly got the elastic complex variable function solution of infinite plate with the ideal crack, according to the boundary conditions of ideal crack, using stress function method. Williams [3] opened the crack-side singularity study in 1957.

In 1920, Griffith [4] studied glass fracture and proposed the concept of energy release rate before the fragile fracture of the US free wheel, which makes a significant contribution to the fracture mechanics. After this, we have energy release rate criterion. The energy release rate criterion is described as follows, when the length of the crack is expanded unit length, the surface energy of the glass increases due to the increase of free surface area, and the strain energy $G$ released by the unit length of the crack is balanced. When the two equals, crack has extended conditions. 
In the 1950s, the use of brittle and high-strength steel in aeronautical structure required a quantitative analysis of the fracture criteria. Later, Irwin proposed the stress intensity factor $K_{I}$ fracture criterion in 1957.

In 1960, Wells suggested that the open displacement of crack top could be the fracture control parameter on the basis of the experiment. Rice and Hutchinson revealed the $J$ integral and singular field theory of elastic-plastic crack tip in 1968, laying the foundation for elastic-plastic fracture mechanics.

\section{The Development of Fracture Mechanics in China}

In the 20th century, China's fracture mechanics research has made great achievements [5] : crack passivation model, fractal geometry of rock fracture, high-speed dynamic crack propagation, expansion crack tip of the singular field, shell theory of cracked plate with shear deformation, weight function method of fracture mechanics, variational method of three-dimensional stress intensity factor, integral equation method of fracture mechanics, shear plastic toughening mechanism of phase change ceramic, non-equilibrium statistical fracture mechanics and so on.

\section{Basic Theory and Fracture Criterion}

Fracture mechanics includes: linear elastic fracture mechanics, elastic-plastic fracture mechanics, rigid plastic fracture mechanics, viscoelastic fracture mechanics, fracture dynamics, composite fracture mechanics and other branches. Three of the most classic parts are: linear elastic fracture mechanics, elastic-plastic fracture mechanics and fracture dynamics.

\section{Linear Elastic Fracture Mechanics}

There are two criteria for linear elastic fracture mechanics: $G$ criterion and $K$ criterion.

(1) $G$ criterion

$G$ criterion is strain energy release rate criterion, and the function is

$$
G_{I}=G_{I C},
$$

where $G_{I}$ is the strain energy release rate. Subtracting load potential energy from strain energy of the object, we get the total energy of the system and find the partial crack cross section of it. Then taking a negative value, strain energy release rate $G_{I}$ is obtained. $G_{I C}$ is the critical strain energy release rate, also known as fracture toughness, is determined by the fracture test of specimen with cracks.

Griffith theory, the study of crack propagation situation of central crack's one-way stretch in infinite plate was originally promoted in non-ideal brittle materials. Irwin and Orowan improved it to be used for non-ideal mildly plastic brittle materials. Irwin suggested that Griffith theory could be used for engineering quasi-brittle materials with the condition that the size of crack tip plastic deformation area is much smaller than the crack length or other feature size.

Although the scope of application in Griffith theory is limited, it is the first time in history of science that considering the characteristic size of the defect in material strength and toughness calculation. At the same time, Griffith suggested that the surface of the solid for the ideal brittle material had ability to resist fracture.

(2) $K$ criterion

The stress intensity factor $K_{I}$ can also be used as a mechanical parameter for the crack propagation criterion. So, we have the two equations:

$$
K_{I}=K_{I C}, K_{I}^{2}=E G_{I}
$$


in which, $E$ is Young's modulus, $K_{I C}$ is critical stress intensity factor. Normally, we can check the reference table to obtain $K_{I C}$, but in some important cases, direct measurements must be take to get $K_{I C}$. In fact, direct tests must meet the following conditions,

1)The numerical results or formulas of sufficient precise stress intensity factors under given load and support conditions are known.

2)The critical value of load corresponding to the initial extension can be measured by direct loading test of the crack body.

3)During the whole process of loading, the effective conditions of the linear fracture mechanics and other tests are met.

The use value of stress intensity factor criterion is very high, but its physical meaning is not clear, which needs further studies. The specific value of $K_{I}$ can be found in the manual [6]. In general, the stress intensity factor can always be written as

$$
K_{I}=F \sigma \sqrt{\pi a} .
$$

In Eq.(3), $\sigma$ is the nominal stress for a certain sense, $a$ is the crack half length. $F$,with dimension of 1 , is the shape factor, depending on the geometry of the specimen and the crack. In the stress intensity factor table, the shape factor is commonly given by fitted analytic expressions or graphs.

\section{Elastic-plastic Fracture Mechanics}

In the 1960s, after the establishment of linear elastic fracture mechanics criterion, the study of elastic-plastic fracture mechanics began.

(1) COD criterion

In 1965, Wells proposed the COD criterion, in which the opening displacement of the crack tip $\delta$ is the fracture parameter, and the critical value of the opening displacement of the crack $\delta_{C}$ is taken as the fracture criterion.

(2) Conservative integral and $J$ integral

Continuous mechanics proves that there is an surface integral, also called conservation integral. And the integer function is formed by stress component, displacement component and the partial derivative on a certain surface. Under certain conditions, such integrals have the meaning of energy and have a conservation property independent of integral surface selection. For the crack body, this kind of integral is related to stress field of the crack front and the characteristic of displacement field. The experiment has already proved that the integral has critical properties for the propagation of the crack in the material.

The modern continuous mechanics proves that there are three basic conservation integrals: $E$ type integral, $L$ type integral, $M$ type integral. The $L$ type integral and $M$ type integral are proposed by Knowles and Sterberg [7]. $E$ type integral is a kind of conservation integrals proposed by Eshelby in 1952 for linear elastic solids.

In 1968, Rice put forward $J$ type integral, in fact, $J$ type integral belongs to $E$ type integral, but the application scope extended from the linear elastic solid to the unloading small deformation of elastic-plastic solid. In the same year, Hutchinson, Rice and Rosegren simplified and analyzed the I type crack problem of power-hardening material with the full theory of elastoplasticity, and obtained the singularity of the crack tip stress field. The singular stress field at the crack end was characterized by the plastic stress intensity factor $K_{p}$. It is also pointed out that the relationship between the plastic stress intensity factor $K_{p}$ and the applied stress $\sigma$ can be obtained by $J$ type integral. So the $J$ type integral is used as the control parameter to characterize the singular stress field, and the elastic-plastic fracture criterion with the characteristic parameter $J$ integral is established, called HRR theory:

$$
J=J_{C}
$$




\section{Fracture Dynamics}

Fracture dynamics, known as dynamic fracture mechanics, studies those fracture mechanics problems, in which the inertia effect can not be ignored. Fracture dynamics can be divided into two categories.

(1) The dynamic initiation criterion of crack

The crack is stable and the external force changes rapidly with time, such as vibration, shock, fluctuation (blast wave, seismic wave, etc). In this case, we study the beginning of crack propagation.

$K_{I d}$ is related to the loading rat $\sigma$, which is apparently temperature dependent, and here we make it room temperature, so temperature is assumed to be a constant of dynamic fracture properties of the material. While $K_{I}$ is obviously in connection with the crack length, external stress and the time, then this criterion can be expressed as,

$$
K_{I}(a, \sigma, t)=K_{I d}(\sigma) .
$$

(2) Motion crack propagation and crack arrest Criterion

The external force is constant and the crack propagates rapidly in this case. The propagation of the crack is studied, and we commonly call it propagation crack problem or motion crack problem. The criterion for the propagation and arresting of motion crack is as follows,

$$
K_{I}(a, \sigma, t) \leq K_{I D}(a) \text {. }
$$

In Eq.(6), $a$ is the the velocity of the crack, and material constant is recorded as $K_{I D}(a)$, a function of the velocity of the crack. The equation represents the propagation condition of the crack, and the inequality indicates the arresting condition.

The above discussion is limited to the content of linear elastic fracture kinetics, which is currently the relatively mature part of fracture dynamics for practical application.

\section{Testing Standard}

In 1970, American Society for Testing and Materials presented the world's first fracture toughness test standard ASTM E399-70T. In 1980, China promulgated the "Test method for determining ductility fracture toughness of metallic materials by using public resistance curve", the national standard GB2038-80, which is the theoretical and experimental research results of $J$ type integral, based on a large number of trials conducted with 23 kinds of materials for more than 100 groups of nearly a thousand pieces of the tests. In 2011, the United States issued the ASTM E1820-11 $K_{I C}$ test standard and ASTM E399-09e $2 J_{I C}$ test standards, the full name of which are "Standard test method for measurement of fracture Toughness"[8], "Standard test method for linear elastic plane strain fracture toughness $\mathrm{K}_{\mathrm{IC}}$ of metallic materials"[9] respectively.

\section{Conclusions}

Fracture mechanics is increasingly important, as it can predict and estimate the safety of materials and structures. In this paper, some basic theories of linear elastic fracture mechanics, elastic-plastic fracture mechanics and fracture dynamics are introduced around four fracture criteria.

At present, the development of linear elastic fracture mechanics is mature enough, but the physical meaning of the stress intensity factor $K$ in the criterion is still not clear. As for fracture dynamics, the nonlinear materials needs to be further studied.

Several important development trends of Fracture mechanics are as follows: fracture from the project closely integrated with the department of engineering to information intelligent materials, natural (such as rock, etc) and biological materials; In order to meet the development needs, fracture mechanics will face the key fracture and damage problems of efficient power aircraft, new Space vehicles, high-speed rail transit, nuclear energy and other important facilities; And fracture mechanics 
is also of great significance to the structural health monitoring and the structural disasters evolution analysis.

\section{Acknowledgement}

This work was supported by the National Natural Science Foundation of China (Grant Nos. 11472160 and 11672185).

\section{References}

[1] C.E. Inglis, Stresses in a plate due to the presence of cracks and sharp corners, Proc Inst Naval Arch. 1913, 55:219-241.

[2] H.M. Westergaard, Bearing pressures and crack, Appl Mech, ASME Trans. 1939, 6:49-53.

[3] M.L. Williams, On the stress distribution at the base of a stationary crack, Appl Mech, ASME. 1957, 24:109-114.

[4] A.A. Griffith, The phenomena of rupture and flow in solids, Phil Trans. 1920, A221:163-198.

[5] Chinese Society of Mechanics, Mechanical Science Development Report, China Science and Technology Press, Bejing, 2007. (in Chinese)

[6] China Aviation Research Institute, Stress strength factor manual, Science Press, Bejing, 1993. (in Chinese)

[7] J.K. Nowle, E. Sterberg, On a class of conservation laws in linearized and finite elastotatics, Arch Rat Mech Anal. 1972,44(3):187-211.

[8] American Society for Testing and Materials, U.S.,ASTME1820-11, 2011.

[9] American Society for Testing and Materials, U.S., ASTM E399-09e2, 2011. 